\title{
ON $L_{1}$-CONTRACTION FOR SYSTEMS OF CONSERVATION LAWS
}

\author{
JORGE G. S. PATIÑNO
}

(Communicated by Walter Littman)

\begin{abstract}
We prove that for $2 \times 2$, strictly hyperbolic, genuinely nonlinear systems of conservation laws, there is no metric $D$ such that$$
\int_{-\infty}^{\infty} D(u(x, t), c) d x
$$

is a nonincreasing function of time for every weak solution $u, u_{0}( \pm \infty)=c$.
\end{abstract}

For $2 \times 2$, strictly hyperbolic, genuinely nonlinear (cf. [1]) systems of conservation laws it was proved in [2] that there is no metric $D$, compatible with the state space, such that

$$
I_{D}(u, v ; t) \equiv \int_{-\infty}^{\infty} D(u(x, t), v(x, t)) d x
$$

is a nonincreasing function of time for any two weak solutions $u, v$ whose initial conditions agree off a compact set.

In [2] a metric $D$ is compatible with the state space $\Sigma$ if

C1. $D: \Sigma \times \Sigma \rightarrow \mathbf{R}$ is a symmetric function.

C2. $D(u, v)+D(v, w) \geqslant D(u, w) \forall u, v, w \in \Sigma$.

C2. $C_{0}^{-1}|u-v| \leqslant D(u, v) \leqslant C_{0}|u-v| \forall u, v \in \Sigma$ with a uniform constant $C_{0}$.

We generalize and simplify the methods in [2], and this enables us to relax condition $\mathrm{C} 3$.

We wish to point out that relaxing condition $\mathrm{C} 3$ is important since it rules out the use e.g. of entropies or quadratic functions to obtain certain integral decay estimates. It is also interesting to note that the solutions used in the construction of the counterexamples below are the elementary "spikes" used frequently in decay arguments (cf. [3]).

Thus let

$$
u_{t}+(f(u))_{x}=0
$$

be any $2 \times 2$ system, strictly hyperbolic, genuinely nonlinear and without coinciding shock and rarefaction curves on a region $N \subset \mathbf{R}^{2}$. Let $\lambda_{1}(u)$ and $\lambda_{2}(u)$ be the eigenvalues of $d f(u)$ with corresponding eigenvectors $r_{1}(u)$ and $r_{2}(u)$. Let $R_{1}\left(u, u^{*}\right)$

Received by the editors July 10, 1986.

1980 Mathematics Subject Classification (1985 Revision). Primary 35L65, 76N10.

Key words and phrases. Conservation laws, decay of solutions, $L_{1}$-dependence.

Work done while the author was a visiting member of the Mathematics Research Center, University of Wisconsin-Madison.

Sponsored by the United States Army under Contract No. DAAG29-80-C-0041. 
and $R_{2}\left(u, u^{*}\right)$ be respectively the integral curves of $r_{1}(u)$ and $r_{2}(u)$ passing through $u^{*} . R_{1}\left(u, u^{*}\right)$ and $R_{2}\left(u, u^{*}\right)$ are called rarefaction curves. Let $S_{1}\left(u, u^{*}\right)$ and $S_{2}\left(u, u^{*}\right)$ be the curves of states that can be joined by, respectively, a 1-shock and 2 -shock to the right of $u^{*}$. These are called shock curves. Given a state $u^{*}$ on $N$, shock and rarefaction curves exist locally [1].

We then have the following theorem.

THEOREM 1. Let $u$ and $v$ be weak solutions of (2) whose initial conditions agree off a compact set. Then there exists no metric $D$, which is symmetric, such that $I_{D}(u, v ; t)$ is a nonincreasing function of time.

Proof. Take any states $u_{L}, u_{R}, \bar{u}$, and $\overline{\bar{u}}$ related in the following way (see Figure 1).

(i) $u_{R}$ and $u_{L}$ are joined by a 1 -shock with speed $s_{1}$, with $u_{L}$ on the left.

(ii) $u_{R}$ and $\bar{u}$ are joined by a 1 -rarefaction.

(iii) $\bar{u}$ and $u_{L}$ are joined by a 2-rarefaction.

(iv) $\overline{\bar{u}}$ and $u_{L}$ are joined by a 2 -rarefaction.

(v) $\overline{\bar{u}}$ and $u_{L}$ are joined by a 1 -rarefaction.

(We assume here that $\lambda_{2}(u)$ increases from $\bar{u}$ to $u_{L}$. The case where $\lambda_{2}(u)$ decreases from $\bar{u}$ to $u_{I}$ is discussed below.)

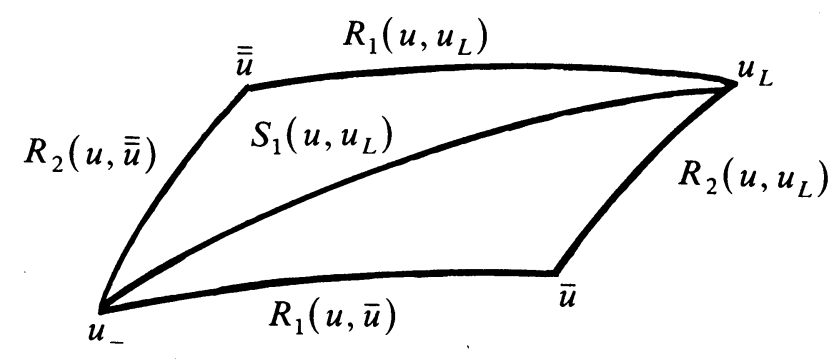

Figure 1

The system (2) with initial condition

$$
u(x, 0)=\left\{\begin{array}{ll}
u_{R} & \text { if } 0<x<\left(s_{1}-\lambda_{1}\left(u_{R}\right)\right) T, \\
u_{L} & \text { otherwise }
\end{array},\right.
$$

has, for $t \leqslant T$, the solution $u$ shown in Figure 2 .

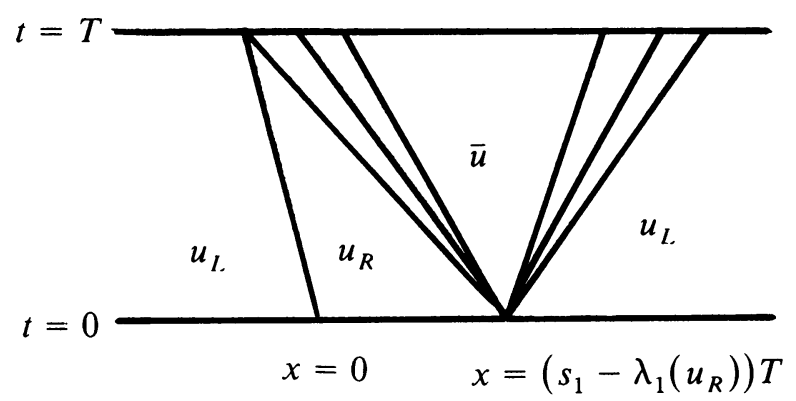

Figure 2. A line denotes a shock and a fan denotes a rarefaction. 
Then

$$
I_{D}\left(u, u_{L} ; 0\right)=D\left(u_{R}, u_{L}\right)\left(s_{1}-\lambda_{1}\left(u_{R}\right)\right) T
$$

and

$$
\begin{aligned}
I_{D}\left(u, u_{L} ; T\right)= & T \int_{\lambda_{1}\left(u_{R}\right)}^{\lambda_{1}(\bar{u})} D\left(\mu(\lambda), u_{L}\right) d \lambda+D\left(u_{L}, \bar{u}\right)\left(\lambda_{2}(\bar{u})-\lambda_{1}(\bar{u})\right) T \\
& +T \int_{\lambda_{2}(\bar{u})}^{\lambda_{2}\left(u_{L}\right)} D\left(\nu(\lambda), u_{L}\right) d \lambda
\end{aligned}
$$

where $\mu(\lambda)$ and $\nu(\lambda)$ denote parametrizations of $R_{1}(u, \bar{u})$ with respect to $\lambda_{1}$ and of $R_{2}\left(u, u_{L}\right)$ with respect to $\lambda_{2}$, respectively.

Now, with $u_{R}, u_{L}$, and $\bar{u}$ denoting the same states as in Figure 1, consider the following initial condition:

$$
v(x, 0)= \begin{cases}u_{L} & \text { if } 0<x<\left(\lambda_{2}\left(u_{L}\right)-s_{1}\right) T \\ u_{R} & \text { otherwise }\end{cases}
$$

The solution $v$ of this problem, for $t \leqslant T$, is given by the waves in Figure 3 .

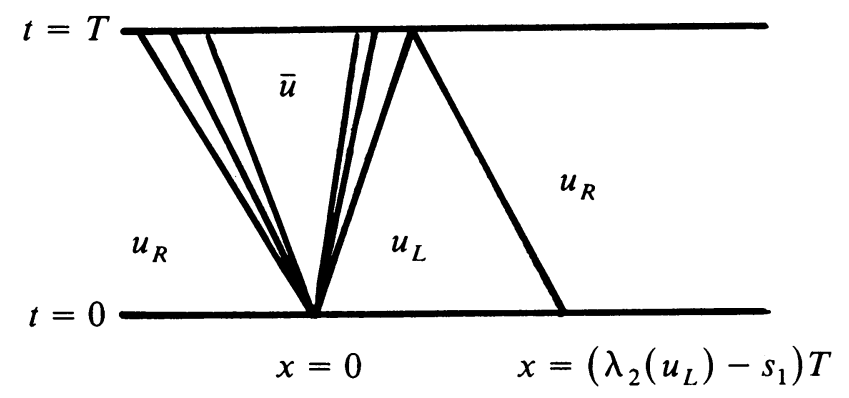

FIgURE 3

Then

$$
I_{D}\left(v, u_{R} ; 0\right)=D\left(u_{L}, u_{R}\right)\left(\lambda_{2}\left(u_{L}\right)-s_{1}\right) T
$$

and

$$
\begin{aligned}
I_{D}\left(v, u_{R} ; T\right)= & T \int_{\lambda_{1}\left(u_{R}\right)}^{\lambda_{1}(\bar{u})} D\left(\mu(\lambda), u_{R}\right) d \lambda+T \int_{\lambda_{2}(\bar{u})}^{\lambda_{2}\left(u_{I}\right)} D\left(\nu(\lambda), u_{R}\right) d \lambda \\
& +D\left(\bar{u}, u_{R}\right)\left(\lambda_{2}(\bar{u})-\lambda_{1}(\bar{u})\right) T .
\end{aligned}
$$

To prove the theorem by contradiction, assume now that

$$
I_{D}\left(v, u_{R} ; T\right)+I_{D}\left(u, u_{L} ; T\right) \leqslant I_{D}\left(v, u_{R} ; 0\right)+I_{D}\left(u, u_{L} ; 0\right) .
$$

Thus

$$
\begin{aligned}
\int_{\lambda_{1}\left(u_{R}\right)}^{\lambda_{1}(\bar{u})}\left\{D\left(\mu(\lambda), u_{L}\right)+D\left(\mu(\lambda), u_{R}\right)\right\} d \lambda \\
\quad+\int_{\lambda_{2}(\bar{u})}^{\lambda_{2}\left(u_{l}\right)}\left\{D\left(\nu(\lambda), u_{L}\right)+D\left(\nu(\lambda), u_{R}\right)\right\} d \lambda \\
\quad+D\left(u_{L}, \bar{u}\right)\left(\lambda_{2}(\bar{u})-\lambda_{1}(\bar{u})\right)+D\left(\bar{u}, u_{R}\right)\left(\lambda_{2}(\bar{u})-\lambda_{1}(\bar{u})\right) \\
\leqslant \\
\quad D\left(u_{R}, u_{L}\right)\left(s_{1}-\lambda_{1}\left(u_{R}\right)\right)+D\left(u_{L}, u_{R}\right)\left(\lambda_{2}\left(u_{L}\right)-s_{1}\right) .
\end{aligned}
$$


Now, adding and subtracting

$$
D\left(u_{L}, u_{R}\right)\left(\lambda_{1}(\bar{u})-\lambda_{1}\left(u_{R}\right)\right)+D\left(u_{L}, u_{R}\right)\left(\lambda_{2}\left(u_{L}\right)-\lambda_{2}(\bar{u})\right)
$$

we get

$$
\begin{aligned}
& \int_{\lambda_{1}\left(u_{R}\right)}^{\lambda_{1}(\bar{u})}\left\{D\left(\mu(\lambda), u_{L}\right)+D\left(\mu(\lambda), u_{R}\right)-D\left(u_{L}, u_{R}\right)\right\} d \lambda \\
& \quad+\int_{\lambda_{2}(\bar{u})}^{\lambda_{2}\left(u_{l .}\right)}\left\{D\left(\nu(\lambda), u_{L}\right)+D\left(\nu(\lambda), u_{R}\right)-D\left(u_{R}, u_{L}\right)\right\} d \lambda \\
& +\left(D\left(u_{L}, \bar{u}\right)+D\left(\bar{u}, u_{R}\right)-D\left(u_{R}, u_{L}\right)\right)\left(\lambda_{2}(\bar{u})-\lambda_{1}(\bar{u})\right) \leqslant 0 .
\end{aligned}
$$

By the triangle inequality the two integrands and the third line above are positive. Since $\lambda_{1}\left(u_{R}\right)<\lambda(\bar{u})$ and $\lambda_{2}(\bar{u})<\lambda_{2}\left(u_{L}\right)$, equality holds in (4) if and only if equality holds in each of the triangle inequalities, in particular only if

$$
D\left(u_{L}, \bar{u}\right)+D\left(\bar{u}, u_{R}\right)=D\left(u_{R}, u_{L}\right)
$$

A similar construction as above using the initial conditions

$$
u_{0}(x)= \begin{cases}u_{L} & \text { if } 0<x<\lambda_{1}\left(u_{L}\right)-s \\ \overline{\bar{u}} & \text { otherwise }\end{cases}
$$

and

$$
u_{0}^{\prime}(x)= \begin{cases}\overline{\bar{u}} & \text { if } 0<x<\lambda_{2}(v)-\lambda_{1}(v) \\ u_{L} & \text { otherwise }\end{cases}
$$

will, by the same argument, yield

$$
D\left(u_{R}, \overline{\bar{u}}\right)+D\left(u_{R}, u_{L}\right)=D\left(\overline{\bar{u}}, u_{L}\right)
$$

Now, (5) and (6) give

$$
D\left(\overline{\bar{u}}, u_{L}\right)-D\left(\bar{u}, u_{R}\right)=D\left(u_{L}, \bar{u}\right)+D\left(u_{R}, \overline{\bar{u}}\right) .
$$

To see that there is no nondegenerate metric satisfying (7), consider the states $u_{R}$, $u_{L}, \bar{u}$, and $\overline{\bar{u}}$ in the space of Riemann invariants. In that space those states form a rectangle which in the metric $D$ has side lengths $a \equiv D\left(u_{L}, \overline{\bar{u}}\right), b \equiv D\left(\overline{\bar{u}}, u_{R}\right)$, $c \equiv D\left(u_{R}, \bar{u}\right)$, and $d \equiv D\left(\bar{u}, u_{L}\right)($ see Figure 4$)$.

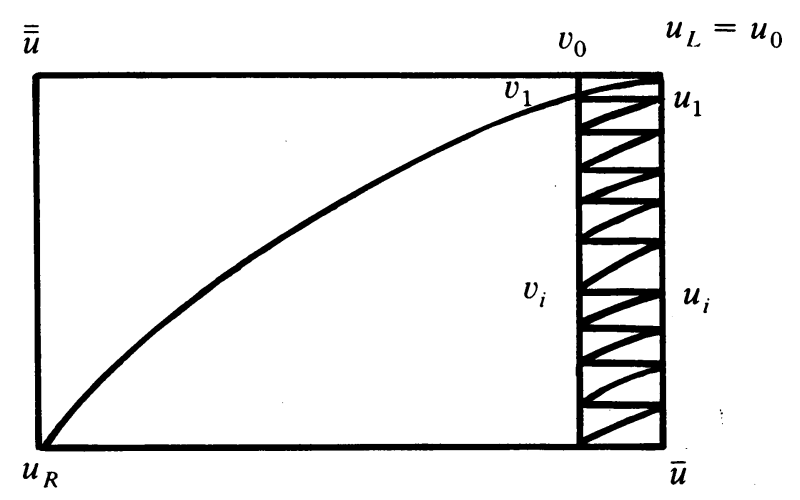

Figure 4 
Then by (7)

$$
a=b+c+d
$$

Now take states $\left\{v_{i}\right\}$ and $\left\{u_{i}\right\}\left(u_{0}=u_{L}\right)$ joined by shock and rarefaction curves as in Figure 4 and let $\alpha_{i} \equiv D\left(v_{i}, u_{i}\right), \beta_{i} \equiv D\left(v_{i+1}, v_{i}\right), \gamma_{i} \equiv D\left(u_{i+1}, u_{i}\right)$.

Since by assumption shock and rarefaction curves do not coincide,

for some finite $N$.

$$
\sum_{i=0}^{N} \gamma_{i}=c<a
$$

Thus

$$
\alpha_{0}=\beta_{0}+\gamma_{0}+\alpha_{1}=\sum_{i=0}^{N}\left(\beta_{i}+\gamma_{i}\right)+\alpha_{N+1} .
$$

Now, if we take $\alpha_{0}<c$,

$$
D\left(u_{N+1}, v_{N+1}\right)=\alpha_{N+1}<-\sum_{i=0}^{N} \beta_{i}<0
$$

which is a contradiction to the fact that $D$ is a positive metric.

In the case where $\lambda_{2}(u)$ decreases from $\bar{u}$ to $u_{L}$ a similar construction using the shock curves $S_{2}(u, \overline{\bar{u}})$ and $S_{2}(u, \hat{u})$ (see Figure 5) and the same initial value problems as above yield conditions similar to (5) and (6) from where the proof would proceed identically.

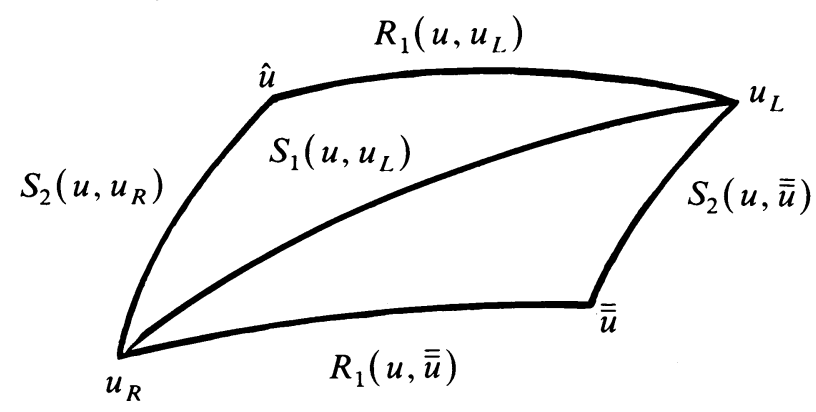

Figure 5

ACKNOWLedgments. I would like to thank Bradley Plohr for reading the first draft and for a good suggestion. I also thank Blake Temple for many discussions on relevant issues. This work was made possible by the financial support of the Conselho Nacional de Pesquisa-CNPq, Brazil, and the Financiadora de Estudos e Projetos-FINEP, Brazil.

\section{REFERENCES}

1. P. D. Lax, Hyperbolic systems of conservation laws. II, Comm. Pure Appl. Math. 19 (1957), 537-566.

2. J. B. Temple, No $L_{1}$-contractive metrics for systems of conservation laws, Trans. Amer. Math. Soc. 288 (1985), 471-480.

3. __ Decay with a rate for noncompactly supported solutions of conservation laws, Trans. Amer. Math. Soc. 298 (1986), 43-82.

Mathematics Research Center, University of Wisconsin - MAdison, Madison, Wisconsin 53706

Current address: Departamento de Matematica, PUC-RJ, Rua Marqués de São Vicente 225, 22453 Rio de Janeiro, Brazil 